\title{
Convergence of solutions of one-dimensional semilinear parabolic equations
}

\author{
By \\ Hiroshi Matano \\ (Received May 5, 1977)
}

\section{Introduction}

The idea of $\omega$-limiting sets borrowed from the theory of dynamical systems has been employed in many authors' works including [1] and [5] to investigate the asymptotic properties of solutions of autonomous parabolic initial-boundary value problems. In the case of parabolic systems, it is well-known that the $\omega$-limiting set of a solution often contains plural elements (in fact infinitely many elements). But, to the best of our knowledge, it has not yet been made clear in the case of single equations whether there exists such a solution as has plural $\omega$-limiting points. The present paper forms part of the answer to this question. That is, we show that the $\omega$-limiting set of any solution contains at most one element providing that the space dimension is one. This result leads to the conclusion that in the case of single onedimensional equations any solution that neither blows up in a finite time nor grows up as $t \rightarrow \infty$ should converge to some equilibrium solution as $t$ tends to infinity.

\section{§1. Notation and Theorems}

Let us consider initial-boundary value problems of the form

$$
\begin{array}{ll}
\text { (1.1a) } & u_{t}=\left\{a(x) u_{x}\right\}_{x}+f(x, u), \quad(0<x<L, 0<t<s), \\
\text { (1.1b) } & u(x, 0)=u_{0}(x), \quad(0 \leqq x \leqq L), \\
\text { (1.1 } \left.c_{0}\right) & \alpha_{0} u(0, t)-\left(1-\alpha_{0}\right) u_{x}(0, t)=\beta_{0}, \quad(0<t<s),
\end{array}
$$$$
\left(1.1 \mathrm{c}_{1}\right) \quad \alpha_{1} u(L, t)+\left(1-\alpha_{1}\right) u_{x}(L, t)=\beta_{1}, \quad(0<t<s) \text {, }
$$

where the coefficient $a(x)$ is positive and sufficiently smooth in the compact interval $[0, L]$ and $\alpha_{i}, \beta_{i}(i=0,1)$ are real constants satisfying $0 \leqq \alpha_{i} \leqq 1(i=0,1)$. The nonlinear term $f$ is also assumed to be a smooth function, say of class $C^{2}$, mapping $[0, L] \times \mathbf{R}$ into $\mathbf{R}$. We choose initial data from the space $C[0, L]$ and consider real-valued classical solutions. A function $v \in C^{1}[0, L] \cap C^{2}(0, L)$ is said to be an 
equilibrium solution of (1.1) if it satisfies the boundary value problem

$$
\begin{gathered}
\left\{a(x) v^{\prime}\right\}^{\prime}+f(x, v)=0, \quad(0<x<L), \\
\alpha_{0} v(0)-\left(1-\alpha_{0}\right) v^{\prime}(0)=\beta_{0}, \\
\alpha_{1} v(L)+\left(1-\alpha_{1}\right) v^{\prime}(L)=\beta_{1} .
\end{gathered}
$$

For any function $\psi \in C[0, L]$, the solution of (1.1) with initial data $u_{0}=\psi$ can always be constructed near $t=0$ by means of the usual successive approximation in the integral equation that is equivalent to (1.1). Here, as in N. Chafee [1], we denote by $(0, s(\psi))$ the largest time interval where the solution of (1.1) with initial data $u_{0}=\psi$ exists. That is to say, we define $s(\psi) \in(0, \infty]$ by

$$
s(\psi)=\sup \{s ; u(x, t, \psi) \text { is continuable to } t=s\},
$$

where $u(x, t, \psi)$ is the solution of (1.1) with initial data $\psi$. By setting $Q(t) \psi=u(\cdot, t$, $\psi)$ for $0 \leqq t<s(\psi)$, we can naturally define an operator $Q(t): C[0, L] \rightarrow C[0, L]$ with parameter $t \geqq 0$. A function $\psi \in C[0, L]$ belongs to the domain of $Q(t)$ if and only if $0 \leqq t<s(\psi)$. It is obvious that $Q(t) \psi$ belongs to $C^{1}[0, L] \cap C^{2}(0, L)$ when $0<t$ $<s(\psi)$ and that

$$
Q(t) \psi=Q\left(t-t^{\prime}\right) Q\left(t^{\prime}\right) \psi
$$

for any $0 \leqq t^{\prime} \leqq t<s(\psi)$.

Definition. Let $\psi \in C[0, L]$ and suppose $s(\psi)=\infty$. The $\omega$-limiting set of $\psi$ is a subset of $C^{1}[0, L] \cap C^{2}(0, L)$ defined as follows:

$$
\Omega(\psi)=\bigcap_{t>0} \text { closure }\{Q(\tau) \psi ; \tau \geqq t\},
$$

where the closure is with respect to the topology of $C^{1}[0, L] \cap C^{2}(0, L)$. We understand that $\Omega(\psi)=\varnothing$ if $s(\psi)<\infty$.

(It should be noted that the $\omega$-limiting set defined above coincides with the one considered in the topology of $C^{1}[0, L]$ or $C[0, L]$. See the author's paper $[5$; Theorem 2.8].)

Now we are ready to formulate our theorems:

Theorem A. The $\omega$-limiting set of any function $\psi \in C[0, L]$ contains at most one element.

Theorem B. For any initial data $\psi \in C[0, L]$, one and only one of the following three holds:

1) $s(\psi)<\infty$ and

$$
\lim _{t \rightarrow s(\psi)}\|Q(t) \psi\|_{L^{\infty}(0, L)}=\infty
$$

that is, the solution blows up in a finite time;

2) $s(\psi)=\infty$ and 


$$
\lim _{t \rightarrow \infty}\|Q(t) \psi\|_{L^{\infty}(0, L)}=\infty
$$

that is, the solution grows up as $t \rightarrow \infty$;

3) $s(\psi)=\infty$ and there exists a solution $v$ of (1.2) such that

$$
\lim _{t \rightarrow \infty} Q(t) \psi=v
$$

in the topology of $C^{1}[0, L] \cap C^{2}(0, L)$.

\section{§2. Derivation of Theorem B from Theorem A}

The following propositions help us to understand that Theorem A implies Theorem B.

Proposition 1. Let $\psi$ belong to $C[0, L]$ and suppose that there exist a sequence $0<t_{1}<t_{2}<\cdots \rightarrow s(\psi)$ and a constant $M>0$ such that

$$
\left\|Q\left(t_{m}\right) \psi\right\|_{L^{\infty}(0, L)}<M
$$

for $m=1,2, \ldots$ Then $s(\psi)=\infty$.

(See [1; Proposition 2.4] and [5; Theorem 2.4i)].)

Proposition 2. Let the same assumption as in Proposition 1 hold. Then $\Omega(\psi)$ is not empty and any element of $\Omega(\psi)$ is a solution of (1.2).

(See [1; Theorem 3.3] and [5; Theorem 2.8].) It is just a trivial fact that the condition in Proposition 1 is satisfied conversely if $\psi$ has a nonempty $\omega$-limiting set.

Proposition 3. Let $\Omega(\psi)$ be nonempty and suppose that it contains an isolated point $v$ with respect to the topology of $C^{1}[0, L] \cap C^{2}(0, L)$. Then $\Omega(\psi)=\{v\}$ and

$$
\lim _{t \rightarrow \infty} Q(t) \psi=v \text { in } C^{1}[0, L] \cap C^{2}(0, L) .
$$

(See [5; Corollary 2.11].)

It is now easy to establish Theorem B with the aid of Theorem A. In fact, due to Proposition 3 and Theorem $\mathrm{A}$, the nonemptiness of $\Omega(\psi)$ implies the convergence of $Q(t) \psi$ to some function as $t \rightarrow \infty$. So the assertion of Theorem B follows immediately if Proposition 1 and 2 are also taken into account.

\section{§3. Proof of Theorem A}

Take an arbitrary function $\psi \in C[0, L]$ that has a nonempty $\omega$-limiting set $\Omega(\psi)$ (so necessarily $s(\psi)=\infty$ ) and fix it. What we have to show is that $\Omega(\psi)$ has just one element. Hereafter, for simplicity, we write $u(\cdot, t)$ instead of writing $Q(t) \psi$. Let us begin with a few lemmas.

Lemma 1. Suppose that $\Omega(\psi)$ contains plural elements and let $v_{1}$ be one of 
them. Then there exists a $t_{0}>0$ such that the zeroes of $u\left(x, t_{0}\right)-v_{1}(x)$ in $[0, L]$ are finite in number.

Proof. Let $v_{2}$ be another element of $\Omega(\psi)$. As $v_{1}$ and $v_{2}$ are distinct solutions of the ordinary differential equation

$$
\left\{a(x) v^{\prime}\right\}^{\prime}+f(x, v)=0, \quad(0<x<L),
$$

the nonnegative function $\left|v_{1}^{\prime}-v_{2}^{\prime}\right|+\left|v_{1}-v_{2}\right|$ never vanishes in the compact interval $[0, L]$. So there exists a $\delta>0$ such that

$$
\left|v_{1}^{\prime}(x)-v_{2}^{\prime}(x)\right|+\left|v_{1}(x)-v_{2}(x)\right| \geqq \delta
$$

for $0 \leqq x \leqq L$. On the other hand, since $v_{2}$ belongs to $\Omega(\psi)$ and since the $\omega$-limiting set here is with respect to the topology stronger than that of $C^{1}[0, L]$, there exists a $t_{0}>0$ satisfying

$$
\sup _{0 \leqq x \leqq L}\left\{\left|u_{x}\left(x, t_{0}\right)-v_{2}^{\prime}(x)\right|+\left|u\left(x, t_{0}\right)-v_{2}(x)\right|\right\}<\delta .
$$

Combining these inequalities, we get

$$
\left|u_{x}\left(x, t_{0}\right)-v_{1}^{\prime}(x)\right|+\left|u\left(x, t_{0}\right)-v_{1}(x)\right|>0
$$

for $0 \leqq x \leqq L$. Therefore, $u\left(x, t_{0}\right)-v_{1}(x)$ cannot have infinitely many zeroes in [0, $L]$, for otherwise the left-hand side of (3.1) must vanish at each accumulation point of these zeroes.

Q.E.D.

The following lemmas need some notation: For each element $v \in \Omega(\psi)$ we set

$$
\begin{aligned}
& A^{+}(v)=\{(x, t) \in[0, L] \times(0, \infty) ; u(x, t)>v(x)\}, \\
& A^{-}(v)=\{(x, t) \in[0, L] \times(0, \infty) ; u(x, t)<v(x)\},
\end{aligned}
$$

and for each $t>0$ we put

$$
\begin{aligned}
& \Sigma_{t}=[0, L] \times[t, \infty), \\
& l_{t}=[0, L] \times\{t\} .
\end{aligned}
$$

Lemma 2. Let $v \in \Omega(\psi)$ and let $C$ be any connected component of $A^{+}(v)$ $\cap \Sigma_{t_{0}}$ in the xt-plane, where $t_{0}>0$. Then $C \cap l_{t_{0}} \neq \emptyset$. The same assertion holds for any connected component of $A^{-}(v) \cap \Sigma_{t_{0}}$.

Proof. We shall prove only the former half, for the latter can be shown just likewise.

First, note that $w=u-v$ satisfies the equation

$$
w_{t}=\left\{a w_{x}\right\}_{x}+f_{u}(x, \xi(x, t)) w
$$

in $(0, L) \times(0, \infty)$, where $v(x) \underset{(\geqq)}{\leqq} \xi(x, t) \underset{(\geqq)}{\leqq} u(x, t)$, and also the boundary conditions

$$
\alpha_{0} w-\left(1-\alpha_{0}\right) w_{x}=0, \quad(x=0, t>0),
$$




$$
\alpha_{1} w+\left(1-\alpha_{1}\right) w_{x}=0, \quad(x=L, t>0) .
$$

Now suppose $C \cap l_{t_{0}}=\emptyset$. Then the function $w$ vanishes along the boundary $\partial C$ of $C$ except on the half lines $\{0\} \times\left(t_{0}, \infty\right)$ and $\{L\} \times\left(t_{0}, \infty\right)$. So, dividing $\partial C$ into three parts $\Gamma_{0}=C \cap\left(\{0\} \times\left(t_{0}, \infty\right)\right), \Gamma_{1}=C \cap\left(\{L\} \times\left(t_{0}, \infty\right)\right)$ and $\Gamma_{2}=\partial C \backslash\left(\Gamma_{0} \cup \Gamma_{1}\right)$, we have

$$
\begin{array}{ll}
\alpha_{0} w+\left(1-\alpha_{0}\right) \frac{\partial w}{\partial n}=0 & \text { on } \Gamma_{0}, \\
\alpha_{1} w+\left(1-\alpha_{1}\right) \frac{\partial w}{\partial n}=0 & \text { on } \Gamma_{1}, \\
w=0 \quad \text { on } \Gamma_{2}, &
\end{array}
$$

where $\partial / \partial n$ denotes the outer normal derivative to $\Gamma_{i} \subset \partial C(i=0,1)$. In the interior of $C$ the equation (3.2) is satisfied. Combining (3.2) and (3.3), and then applying the maximum principle in the usual manner (after a suitable change of the unknown), we obtain

$$
w=0 \text { in } C
$$

(cf. [6; the proof of Theorem 8 in Chap. 3]), which, however, clearly contradicts the relation $C \subset A^{+}(v)$. Thus our supposition $C \cap l_{t_{0}}=\emptyset$ has turned out false.

Q.E.D.

Lemma 3. Suppose that $\Omega(\psi)$ contains plural elements and let $v$ be one of them. Then there exists a $t^{*}>0$ such that $u(0, t)-v(0)$ never changes sign in $\left[t^{*}\right.$, $\infty)$ (that is, $u(0, t)-v(0) \geqq 0$ for $t^{*} \leqq t<\infty$ or $u(0, t)-v(0) \leqq 0$ for $\left.t^{*} \leqq t<\infty\right)$. The same assertion holds for $u_{x}(0, t)-v^{\prime}(0)$.

Proof. First we prove the former part. Let $t_{0}$ be as in Lemma 1. In order to verify the assertion, it is sufficient to show that $u(0, t)-v(0)$ does not change sign infinitely many times in the interval $\left[t_{0}, \infty\right)$.

Suppose that $u(0, t)-v(0)$ changes sign infinitely many times in $\left[t_{0}, \infty\right)$. Then there exists a sequence $t_{0}<t_{1}<t_{2}<\cdots$ such that

$$
\begin{aligned}
& u\left(0, t_{2 k-1}\right)-v(0)>0, \\
& u\left(0, t_{2 k}\right)-v(0)<0,
\end{aligned}
$$

for $k=1,2, \ldots$. It follows from Lemma 2 that each point $P_{2 k-1}=\left(0, t_{2 k-1}\right)$ is connected with some point $Q_{2 k-1} \in A^{+}(v) \cap l_{t_{0}}$ by a curve $C_{2 k-1} \subset A^{+}(v) \cap \Sigma_{t_{0}}$ and that each point $P_{2 k}=\left(0, t_{2 k}\right)$ with some point $Q_{2 k} \in A^{-}(v) \cap l_{t_{0}}$ by a curve $C_{2 k} \subset A^{-}(v)$ $\cap \Sigma_{t_{0}}$. Since the curves $C_{2 k-1}$ and $C_{2 k^{\prime}}\left(k, k^{\prime} \in \mathbf{N}\right)$ cannot intersect each other, the points $Q_{1}, Q_{2}, Q_{3}, \cdots$ are lined on the segment $l_{t_{0}}$ in this order. This means that $u\left(x, t_{0}\right)-v(x)$ changes sign infinitely many times in $[0, L]$, a contradiction to the fact that $u\left(x, t_{0}\right)-v(x)$ has at most finitely many zeroes in $[0, L]$.

Secondly, the proof of the latter part. We only consider the case where $\alpha_{0}=1$, 
for otherwise the latter follows directly from the former by virtue of the boundary condition for $w=u-v$. Let $t_{0}$ be as above and suppose that there exists a sequence $t_{0}<\tilde{t}_{1}<\tilde{t}_{2}<\cdots$ such that

$$
\begin{aligned}
& u_{x}\left(0, z_{2 k-1}\right)-v^{\prime}(0)>0, \\
& u_{x}\left(0, z_{2 k}\right)-v^{\prime}(0)<0,
\end{aligned}
$$

for $k=1,2, \ldots$. Since $u-v$ vanishes on the half-line $\{0\} \times(0, \infty)$, there exist numbers $x_{1}, x_{2}, x_{3}, \cdots \in(0, L)$ such that each segment $\left(0, x_{2 k-1}\right] \times\left\{\tilde{t}_{2 k-1}\right\}$ is contained in $A^{+}(v)$ and that $\left(0, x_{2 k}\right] \times\left\{\mathfrak{t}_{2 k}\right\}$ is contained in $A^{-}(v)$. Now connect each of the points $\left(x_{2 k-1}, \tilde{t}_{2 k-1}\right)(k=1,2, \ldots)$ with some point $\widetilde{Q}_{2 k-1} \in A^{+}(v) \cap l_{t_{0}}$ by a curve wholly contained in $A^{+}(v) \cap \Sigma_{t_{0}}$. Combining this curve and the segment $\left[0, x_{2 k-1}\right] \times$ $\left\{\tilde{t}_{2 k-1}\right\}$, and denoting it by $\tilde{C}_{2 k-1}$, we see that $\tilde{C}_{2 k-1}$ connects the point $\tilde{P}_{2 k-1}$ $=\left(0, \tilde{t}_{2 k-1}\right)$ with $\widetilde{Q}_{2 k-1}$ and is contained in $A^{+}(v) \cap \Sigma_{t_{0}}$ except for the end point $\widetilde{P}_{2 k-1}$. Similarly, each point $\widetilde{P}_{2 k}=\left(0, \tilde{t}_{2 k}\right)$ can be connected with some point $\widetilde{Q}_{2 k}$ $\in A^{-}(v) \cap l_{t_{0}}$ by a curve $\tilde{C}_{2 k}$ which is contained in $A^{-}(v) \cap \Sigma_{t_{0}}$ except for the end point $\widetilde{P}_{2 k}$. As $\widetilde{C}_{2 k-1}$ and $\widetilde{C}_{2 k^{\prime}}\left(k, k^{\prime} \in \mathbf{N}\right)$ cannot intersect each other, the points $\widetilde{Q}_{1}, \widetilde{Q}_{2}$, $\widetilde{Q}_{3}, \cdots$ must be lined on $l_{t_{0}}$ in this order, and so we get a contradiction just as above.

Q.E.D.

Proof of Theorem A. By virtue of Proposition 3, what we have to show is that $\Omega(\psi)$ contains an isolated point. Suppose that it contains no isolated point. As $\Omega(\psi)$ is assumed to be nonempty, it must contain infinitely many elements. Let $v_{1}, v_{2}$ and $v_{3}$ be distinct elements of it. Here we have two cases, the case where $0 \leqq \alpha_{0}<1$ and the case where $\alpha_{0}=1$.

Case 1. Let $0 \leqq \alpha_{0}<1$. Since $v_{1}, v_{2}$ and $v_{3}$ are distinct solutions of the ordinary differential equation

$$
\begin{aligned}
& \left\{a(x) v^{\prime}\right\}^{\prime}+f(x, v)=0, \quad(0<x<L), \\
& v^{\prime}(0)=-\frac{\alpha_{0}}{1-\alpha_{0}} v(0),
\end{aligned}
$$

the values $v_{1}(0), v_{2}(0)$ and $v_{3}(0)$ are different from one another. Without loss of generality we can assume $v_{1}(0)<v_{2}(0)<v_{3}(0)$. Well, from Lemma 3 it follows that there exists a $t^{*}>0$ such that $u(0, t)-v_{2}(0)$ does not change sign in $\left[t^{*}, \infty\right)$. But it obviously contradicts the fact that both $v_{1}$ and $v_{3}$ are elements of $\Omega(\psi)$.

Case 2. Let $\alpha_{0}=1$. In this case $v_{1}^{\prime}(0), v_{2}^{\prime}(0)$ and $v_{3}^{\prime}(0)$ are different from one another. So, using the latter part of Lemma 3 and arguing as above, we easily get a contradiction.

Thus, in either case, our supposition that $\Omega(\psi)$ contains no isolated point has turned out false.

Q.E.D. 


\section{Acknowledgment}

The author expresses his hearty thanks to Professor Masaya Yamaguti and Professor Masayasu Mimura for their stimulating suggestions.

\section{Research Institute for Mathematical Sciences, KYOTO UNIVERSITY}

\section{References}

[1] Chafee, N., Asymptotic behavior for solutions of a one-dimensional parabolic equation with homogeneous Neumann boundary conditions, J. Differential Equations, 18 (1975), 111-134.

[2] Friedman, A., Partial differential equations of parabolic type, Prentice-Hall, 1963.

[ 3 ] Itô, S., Fundamental solutions of parabolic differential equations and boundary value problems, Japan J. Math., 27 (1957), 55-102.

[4] —_ A remark on my paper "A boundary value problem of partial differential equations of parabolic type" in Duke Mathematical Journal, Proc. Japan Acad., 34 (1958), 463-465.

[5] Matano, H., Asymptotic behavior and stability of solutions of semilinear diffusion equations, Publ. Res. Inst. Math. Sci., to appear.

[6] Protter, M. H. and Weinberger, H.F., Maximum principles in differential equations, Prentice-Hall, 1967. 